Abstract 83 Table 1 Characteristics of patients on 2 and 6-week pathways

\begin{tabular}{|c|c|c|c|c|}
\hline & $\begin{array}{l}\text { All Patients } \\
(n=1013)\end{array}$ & $\begin{array}{c}\text { 2-week, } \\
n=544(54 \%)\end{array}$ & $\begin{array}{c}\text { 6-week, } \\
n=469(46 \%)\end{array}$ & $\begin{array}{c}\text { p Value } \\
\text { (2 vs 6-week pathway) }\end{array}$ \\
\hline Mean Age, \pm SD & $79 \pm 9$ & $79 \pm 10$ & $78 \pm 9$ & 0.076 \\
\hline Male, n (\%) & $465(46 \%)$ & $279(52 \%)$ & $186(40 \%)$ & $<0.001$ \\
\hline Mean NTproBNP, \pm SD & $3074 \pm 4077$ & $4896 \pm 4861$ & $961 \pm 411$ & $<0.001$ \\
\hline Mean $\mathrm{Hb}, \pm \mathrm{SD}$ & $127 \pm 18$ & $125 \pm 19$ & $129 \pm 17$ & $<0.001$ \\
\hline Mean $\mathrm{Cr}, \pm \mathrm{SD}$ & $100 \pm 44$ & $109 \pm 50$ & $90 \pm 32$ & $<0.001$ \\
\hline MI, n (\%) & $154(15 \%)$ & $129(24 \%)$ & $25(5 \%)$ & $<0.001$ \\
\hline$A F, n(\%)$ & $485(48 \%)$ & $298(55 \%)$ & $187(40 \%)$ & $<0.001$ \\
\hline HTN, n (\%) & $665(66 \%)$ & $329(60 \%)$ & $336(72 \%)$ & $<0.001$ \\
\hline DM, n (\%) & $218(22 \%)$ & $128(24 \%)$ & 90 (19\%) & 0.094 \\
\hline
\end{tabular}

Abstract 83 Table 2 Characteristics of Heart Failure and Not Heart Failure groups

\begin{tabular}{|l|c|c|c|}
\hline & Heart Failure, $\mathbf{n = 5 4 3}(\mathbf{5 4 \% )}$ & Not Heart Failure, $\mathbf{n = 4 5 4}$ (45\%) & $p$ Value \\
\hline Mean Age, \pm SD & $79 \pm 9$ & $79 \pm 9$ & 0.198 \\
\hline Male, $\mathbf{n}(\%)$ & $270(50 \%)$ & $187(41 \%)$ & $\mathbf{0 . 0 0 7}$ \\
\hline Mean NTproBNP, \pm SD & $4159 \pm 4734$ & $1704 \pm 2400$ & $<\mathbf{0 . 0 0 1}$ \\
\hline Mean Hb, \pm SD & $126 \pm 19$ & $128 \pm 18$ & 0.191 \\
\hline Mean Cr, \pm SD & $103 \pm 42$ & $97 \pm 45$ & $\mathbf{0 . 0 2 9}$ \\
\hline MI, $\mathbf{n}(\%)$ & $96(18 \%)$ & $57(13 \%)$ & $\mathbf{0 . 0 2 5}$ \\
\hline AF, $\mathbf{n}(\%)$ & $295(54 \%)$ & $184(41 \%)$ & $<\mathbf{0 . 0 0 1}$ \\
\hline HTN, $\mathbf{n}(\%)$ & $338(62 \%)$ & $320(70 \%)$ & $\mathbf{0 . 0 0 6}$ \\
\hline DM, $\mathbf{n}(\%)$ & $138(25 \%)$ & $75(17 \%)$ & $<0.001$ \\
\hline
\end{tabular}

The proportion of patients diagnosed with $\mathrm{HF}$ in the 2week pathway was high. As expected, patients referred via the 2-week pathway had significantly higher rates of both mortality and hospitalisation.

This study provides valuable real world data and insight into an important population, which will help inform discussions with patients and shape chronic HF services.

Conflict of Interest None

\section{THE PROGNOSTIC SIGNIFICANCE OF NEUTROPHIL TO LYMPHOCYTE RATIO IN PATIENTS WITH HEART FAILURE WITH REDUCED AND PRESERVED EJECTION FRACTION}

Fraser Curran*, Chim Lang, Colin Palmer, Ify Mordi. University of Dundee

\subsection{6/heartjnl-2019-BCS.82}

Introduction It is increasingly recognised that inflammation plays an important role in the pathogenesis and progression of heart failure (HF) and may have important therapeutic and diagnostic implications. Research has shown that raised inflammatory cytokines are associated with adverse outcomes, however these are not routinely assessed in clinical practice. The aim of this study was to evaluate the association of neutrophil to lymphocyte ratio (NLR), a simple, routinely-measured parameter of inflammatory status, with prognostic outcomes in a cohort of HF patients.

Methods Patients who had survived to 30 days or discharge following an initial HF hospitalisation from the Genetics of Diabetes and Audit Research Tayside Scotland (GoDARTS) study were included. Baseline clinical and demographic data were obtained from electronic health records. Patients with available echocardiography were used to perform a stratified analysis (HFrEF - ejection fraction $<50 \%$, HFpEF 50\%). NLR was calculated from baseline blood results taken nearest the time of initial HF diagnosis (within 90 days). The primary outcome was combined all-cause mortality or HF hospitalisation. Results In total 1,157 patients with HF were included (mean age 73 years, $61.7 \%$ male, $740 \mathrm{HFrEF,} 417 \mathrm{HFpEF}$ ), 847 reached primary outcome (713 deaths and 471 HF admissions). Median follow-up was 769 days. In multivariable analysis (adjusting for blood-pressure, cholesterol, age, gender, diabetes and smoking status) higher NLR at the time of HF diagnosis was significantly associated with the primary outcome (HR 1.04; 95\% CI 1.02-1.05, p<0.001) and the individual endpoints (all-cause mortality HR 1.03; 95\% CI $1.02-$ $1.05, \mathrm{p}<0.001$; HF hospitalisation HR 1.03; 95\% CI 1.01$1.05, \mathrm{p}=0.002)$. When stratified by ejection fraction higher 


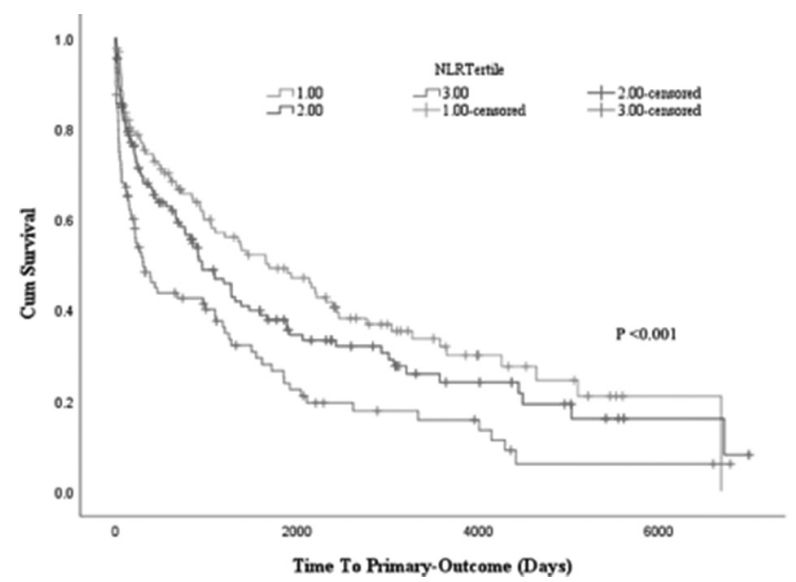

Abstract 84 Figure 1 KM Curve HFpEF Group

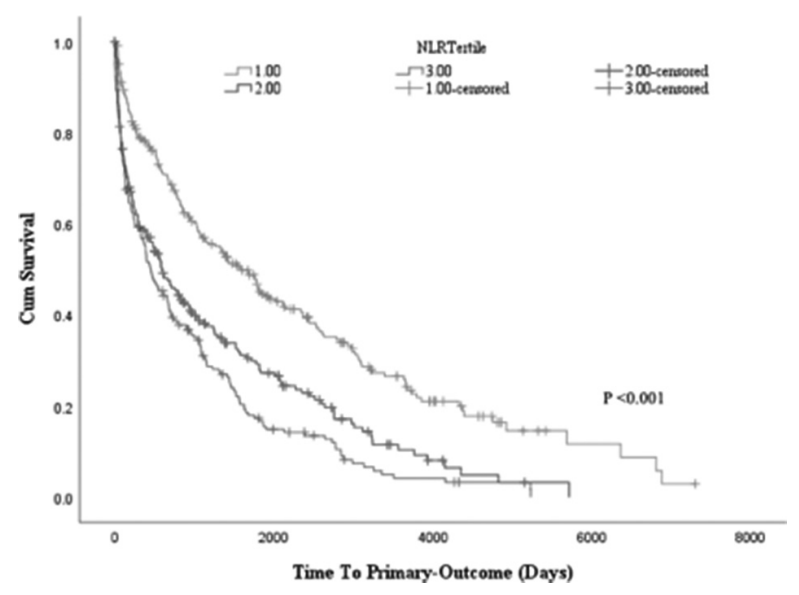

Abstract 84 Figure 2 KM Curve HFrEF Group

NLR at the time of admission was significantly associated with the primary outcome in both HFrEF (HR 1.04; 95\% CI $1.03-1.06, \mathrm{p}<0.001)$ and HFpEF (HR 1.04; 95\% CI 1.011.06, $\mathrm{p}=0.002$ ) and all-cause mortality (HFrEF HR 1.03; 95\% CI 1.02-1.05, p<0.001, HFpEF HR 1.04; 95\% CI 1.02-1.07, $\mathrm{p}=0.01)$. NLR was only associated with HF hospitalisation alone in HFrEF HR 1.04; 95\% CI 1.02-1.06, $\mathrm{p}<0.001$; HFpEF HR 1.01; 95\% CI 0.96-1.05, p=0.9). Interaction testing showed no significant difference in the association of NLR and the primary outcome (p-value 0.65), or individual end-points (all-cause mortality $\mathrm{p}=0.47$, HF hospitalisation $p=0.22$ ) based on ejection fraction. Results of KaplanMeier (KM) analysis highlighting the association between NLR Tertiles and the primary outcome in HFpEF and HFrEF can be seen in Figure 1. and Figure 2, respectively.

Conclusion We found that NLR, a simple measure of inflammation was significantly associated with prognosis in $\mathrm{HFrEF}$ and HFpEF. These results support the growing consensus that inflammation plays a key role in HF. Future research should focus on comparing the NLR with established markers in HF, and the potential use of anti-inflammatory interventions targeting NLR in the treatment of HF.

Kaplan-Meier curves of the association between NLR Tertiles and the primary outcome in HFpEF and HFrEF:

Conflict of Interest No

\section{VARIATION IN HEART FAILURE SERVICE PROVISION ACROSS THE UK: RESULTS FROM A SURVEY OF 100 SERVICES}

${ }^{1}$ Helena Masters*, ${ }^{2} J o n$ Freeman, 'Samantha Dixon. ${ }^{1}$ Novartis Pharmaceuticals UK Limited;

${ }^{2}$ Synergy Healthcare Research

\subsection{6/heartjnl-2019-BCS.83}

Introduction The latest NICE guidelines for chronic HF, published in 2018, provided updated recommendations on best practice for the diagnosis and management of HF. How far does current service provision meet guideline recommendations and how do services compare across the UK? We carried out a survey to determine the different models of HF services across the UK, to characterise current service provision and understand how HF is currently managed.

Methods Data were collected from HF services across the UK via postal survey or telephone interview between February and May 2018. Questions included in the survey were refined after 11 pilot interviews with lead HF nurses conducted between December 2017 and January 2018.

Results One hundred HF services completed the survey: 56 HF nurses and $44 \mathrm{HF}$ cardiologists provided data for 67 trusts and 1 social enterprise in England, and 5 health boards in Scotland, 4 in Wales and 2 in Northern Ireland. The average population size served by an HF service was $-600,000$ people (range 22,000 to 3.5 million), with $\sim 1600 \mathrm{HF}$ patients under their care (range 60-20,000) and -480 new HF patients (range 12-2000) referred into the service in the last 12 months. Most services saw patients in both hospital and the community $(66 \%)$, while fewer saw only hospital $(18 \%)$ or community $(16 \%)$ patients. Care in the community was provided by the majority of services for HFrEF (95\%) and for end-of-life care, post-MI HF and HFmrEF (-80\% each), while only $53 \%$ of services saw patients with HFpEF in the community. The number of HF nurses varied widely between $\mathrm{HF}$ services: 1-2 nurses (26\%); 3-4 (22\%); 5-6 (24\%) and 7 (28\%). Almost one quarter of HF services (24\%) had no administrative support, while $15 \%$ did not have a consultant with an interest in HF in their area. Only 1 in 4 services (27\%) had a pharmacist, while even fewer had a mental health professional (14\%) working within their team.

Conclusion HF service provision across the UK is highly variable. Around 1 in 7 services did not have a consultant or a lead physician with an interest in HF, highlighting a deficit between NICE guideline recommendations and clinical practice. The lack of administrative support in a quarter of HF services is of concern considering the large number of patients these services support. Resourcing issues may account for the type of patients HF services see, as considerably fewer services offer care in the community to patients with HFpEF compared with other types of HF.

Funding This study was supported by Novartis. Writing assistance was provided by Hollie Robinson, $\mathrm{PhD}$, of Complete HealthVizion, funded by Novartis.

Conflict of Interest Employee of Novartis 